\title{
Organ-specific microbial signatures in obesity and type 2 diabetes mellitus
}

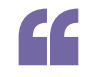

These data

provide

evidence for

the presence

of selective

plasma

and tissue

microbial

signatures in

individuals with

severe obesity

5

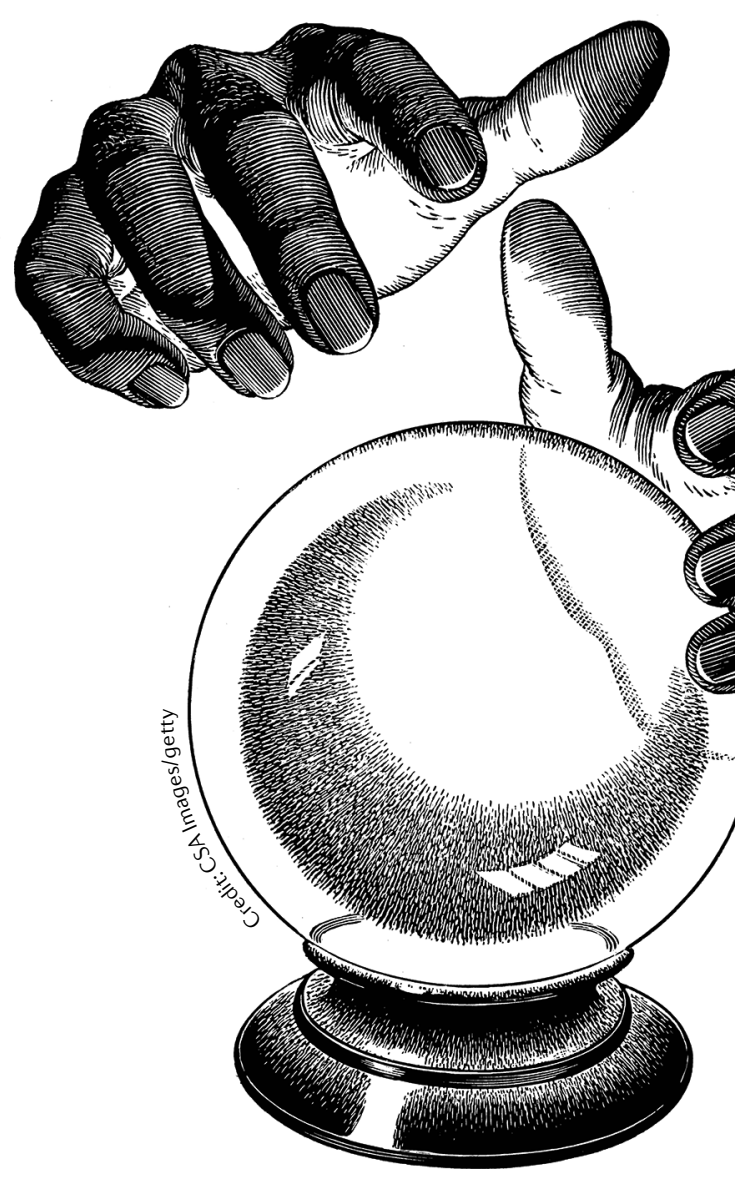

have shed doubt on data that show microbial signatures in the blood or adipose tissue of people with obesity and T2DM. In the present study, however, corresponding author André Marette and colleagues overcame the experimental obstacles by diligently controlling for contamination and including a large number of control samples, which reduced the risk of reporting false-positive results.

"The gut microbiome is a well-established determinant of metabolic health and disease, but very little was known about the potential for tissue microbiomes in modulating metabolic homeostasis," explains corresponding author André Marette. The team therefore investigated whether bacterial signatures could be detected in people with severe obesity. "We hypothesized that patients with severe obesity would have a leaky gut that is likely to be more permeable to bacteria or bacterial components, which can reach the circulation and metabolic tissues such as the liver and fat depots," adds Marette. Marette and colleagues used $16 \mathrm{~S}$ rRNA sequencing to investigate bacterial signatures in plasma, liver and three visceral or subcutaneous adipose tissue depots (specifically, mesenteric, omental and subcutaneous adipose tissue). "We wanted to

look at specific inter-organ signatures and compare the signatures from individuals with obesity with or without T2DM in order to determine the effect of dysglycaemia," explains Marette. The cohort included 10 men and 30 women. Five men and 15 women $(n=20)$ had normal glucose tolerance $\left(\mathrm{HbA}_{1 \mathrm{c}}<5.7 \%\right.$ or fasting plasma glucose $<6.1 \mathrm{mM}$ ), whereas 5 men and 15 women $(n=20)$ had T2DM (fasting plasma glucose

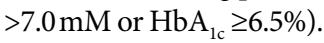

The team found that individuals with obesity have distinct bacterial signatures in the plasma and liver, as well as mesenteric, omental and subcutaneous adipose tissue. Furthermore, the authors were able to identify bacterial signatures that were associated with the presence of T2DM. These bacterial signatures were most pronounced in mesenteric adipose tissue, in which individuals with T2DM displayed reduced bacterial diversity concomitant with fewer Gram-positive bacteria, as opposed to increased levels of typically opportunistic Gram-negative Enterobacteriaceae. "Plasma samples of individuals with T2DM were similarly enriched in Enterobacteriaceae, such as Escherichia-Shigella," adds Marette. "These data provide evidence for the presence of selective plasma and tissue microbial signatures in individuals with severe obesity and identifies new potential microbial targets and biomarkers of T2DM."

The team now plan to identify the physiological traits that predispose individuals to bacterial translocation. "We would also like to investigate to what extent live bacteria or the bacterial components that are found in metabolically relevant tissues promote or respond to T2DM status," adds Marette. The overarching aim of this work is to identify bacteria or bacterial components that preserve glucose regulation in individuals with both normoglycaemia and morbid obesity in order to identify new potential microbial targets and biomarkers of T2DM.

Alan Morris

ORIGINAL ARTICLE Anhê, F. F. et al. Type 2 diabetes influences bacterial tissue compartmentalisation in human obesity. Nature Metabolism 2, 233-242 (2020) 